\title{
Functional Characterization of Elements in a Human U6 Small Nuclear RNA Gene Distal Control Region
}

\author{
DEBORAH A. DANZEISER, OLGUI URSO, AND GARY R. KUNKEL* \\ Department of Biochemistry and Biophysics, Texas A\&M University, College Station, Texas 77843-2128
}

Received 19 January 1993/Returned for modification 8 March 1993/Accepted 17 May 1993

\begin{abstract}
The promoters of vertebrate U6 small nuclear RNA genes contain a distal control region whose presence results in at least an eightfold level of transcriptional activation in vivo. Previous transfection experiments have demonstrated that most of the distal control region of a human $\mathrm{U} 6$ gene resides in a restriction fragment located from -244 to -149 relative to the transcriptional start site. Three octamer-related motifs that bind recombinant Oct-1 transcription factor in vitro exist in this segment of DNA. However, transfection of human 293 cells with various plasmid templates in which these Oct-1 binding sites had been disrupted individually or in combination showed that only the consensus octamer motif located between positions -221 to -214 was functional. Even so, the consensus octamer motif mutant template was expressed at only a moderately reduced level relative to the wild-type promoter. When another octamer-related sequence located nearby, one that did not bind Oct-1 in vitro, was disrupted along with the perfect octamer site, expression was reduced fivefold in transfected cells. A factor that binds this functional, nonconsensus octamer site (NONOCT) was detected in crude cellular extracts. However, the NONOCT sequence was not essential for activation, since its disruption caused only a $40 \%$ reduction in $\mathrm{U} 6$ gene expression, and mutagenesis to convert the NONOCT sequence to a consensus octamer motif restored wild-type expression. Furthermore, in vitro transcription of a human U6 proximal promoter joined to a single copy of the octamer motif was stimulated by the addition of recombinant Oct-1 protein.
\end{abstract}

Vertebrate U6 small nuclear RNA (snRNA) genes are prototypes of the subclass of RNA polymerase III (Pol III)-transcribed genes that contain upstream promoters and no intragenic control region $(10,20,29)$. The organization of these promoters is remarkably similar to those of genes transcribed by RNA Pol II. A general division of the vertebrate U6 gene promoter differentiates (i) a proximal region within $70 \mathrm{bp}$ of the transcriptional start site consisting of an snRNA gene proximal sequence element (PSE) and a TATA element from (ii) a distal region greater than $200 \mathrm{bp}$ upstream that usually contains an octamer motif. Similarly, promoters of the vertebrate Pol II snRNA genes (e.g., U1 and U2 genes) contain PSE and octamer motifs in nearly identical locations but lack recognizable TATA boxes (9).

snRNA promoters are among the most powerful ones in rapidly growing cells. It has been estimated that a human U1 or U2 transcript destined for completion must be initiated every 2 to $4 \mathrm{~s}(3,9)$. The distal region plays a major role in the efficiency of snRNA promoters, accounting for a 5- to 100-fold level of activation of both Pol II- and Pol IIImediated transcription in transfected mammalian cells or injected Xenopus oocytes $(3,4,6,17,19,22,27,40)$. Enhancers of snRNA genes and mRNA genes are not completely interchangeable, suggesting that the mechanism of snRNA gene activation may be distinct $(8,11,43,44)$. Many Pol II-transcribed snRNA gene distal regions have been dissected and found to be composed of an octamer element and a second sequence element in close proximity. Some of the second sequence elements that have been identified include Sp1 binding sites $(2,14,45)$, SPH motifs (7, $35,36,50$ ), an AP-2 binding site (48), a cyclic AMP (cAMP) response element $(48)$, and a CCAAT motif $(1,38)$. In the

\footnotetext{
*Corresponding author. Electronic mail address: KUNKEL@ TAMBIGRF.
}

case of the human U2 snRNA distal region, Oct-1 protein and Spl may interact (15).

The organization of vertebrate U6 gene distal regions has not been well studied. The consensus octamer motif is bound by a protein in crude nuclear extracts $(4,6)$, but the potential for other sequence elements has been examined only for the Xenopus U6 gene, in which case an Sp1 binding site is involved (25). Results from transfection experiments demonstrated that the distal region of the Pol III-transcribed human 7SK gene, whose promoter is closely related to those of vertebrate U6 genes $(20,28,29)$, contains a CACCC-box element (19). A role for the nonconsensus octamer elements in the 7SK gene distal region is not certain $(18,19)$, although these sites are bound by purified octamer binding factors in vitro (30). However, the potential for octamer factor function of the 7SK promoter is clear, since purified Oct-1 and Oct-2 stimulate Pol III-mediated transcription in vitro (30, 31 ), and the presence of a consensus octamer motif acts as a potent activator sequence in vivo $(18,31)$. Interestingly, a single octamer motif is sufficient for stimulation of this Pol III small RNA promoter, whereas the enhancers of Pol II snRNA genes generally are composed of two elements, one of which is an octamer motif.

The lack of an efficient in vitro transcription system for Pol II snRNA gene promoters has delayed progress in understanding the molecular mechanism of this important class of enhancer-like elements. In contrast, in vitro transcription from Pol III snRNA gene promoters has been detected much more easily such that the formation of transcriptional complexes and the roles of purified factors can be studied $(21,26$, $30,34,39,46,47)$. Therefore, we have begun the characterization of a human U6 snRNA gene distal region as a step to delineate the mechanism of snRNA gene activation. We demonstrate that full activation of this U6 promoter in vivo depends on both the consensus octamer motif and a nearby 
element that we refer to as NONOCT. A protein distinct from Oct-1 recognizes the NONOCT element in crude cellular extracts. However, the NONOCT element is not required for efficient stimulation of the human U6 promoter, since wild-type transcriptional levels can be obtained in transfection experiments when the NONOCT sequence is replaced by another perfect octamer motif. Furthermore, in vitro transcription directed by this promoter is stimulated by recombinant Oct-1 (rOct-1) protein with a template containing a single octamer motif.

\section{MATERIALS AND METHODS}

Plasmid constructions. Plasmids containing a human U6 maxigene with a wild-type promoter, a deletion of the $5^{\prime}$ flanking region to position $-148(d l-148)$, and clustered point mutations in the TATA element (TATAMUT), PSE (PSE MUT), and consensus octamer motif (OCTCONMUT; previously called OCTMUT) have been described before (2123). Other plasmids containing mutated sequences were constructed by the same oligonucleotide-directed techniques, using M13mp18 single-stranded DNA templates that included uracil residues (24). Oligodeoxynucleotides used for these constructions were as follows (nucleotide changes from the wild-type promoter sequence are shown in lowercase type): NONOCTMUT (-256 GCAGGAAGAGGGCCT tcTagagATGATTCCTTCATA -221), OCT2MUT (-200 CT GTTAGAGAGATAAggtaccTTAATTTGACTGTAA -165), OCT3MUTWT (-190 GATAATTAGAATTAAccatggTGT AAACACAAAGAT -155), OCT3MUT2 (-190 GATAAggt accTTAAccatggTGTAAACACAAAGAT -155), and CON VROCT [(-252) GAAGAGGGCCTATTTg-CATGATTCC TTCATA -221].

The OCT3MUT2 oligonucleotide was used to disrupt the OCT3 DNA sequence in a template that contained a mutated OCT2 site. The CONVROCT oligonucleotide converted the wild-type NONOCT sequence to a consensus octamer sequence, a change that resulted in the deletion of $1 \mathrm{bp}$. Inserts from recombinant $\mathrm{M} 13 \mathrm{mp} 18$ replicative-form DNAs containing desired mutations were subcloned into the pGEM1 vector (Promega) for use in transfection experiments.

For use in in vitro transcription experiments, the 5' flanking region of the U6 promoter in the U6/(GGAT) $)_{10}$ plasmid (21) was deleted to position -84 by restriction with DraI and EcoRI and ligation into plasmid pGEM3Zf(-) (Promega) that had been restricted with SmaI and EcoRI $\left[d l-84 / \mathrm{U} 6(\mathrm{GGAT})_{10}\right]$. A single consensus octamer motif contained in a double-stranded oligonucleotide (OCTCON; sequence given below) was ligated into the BamHI site of the $d l-84$ construct to yield plasmid +OCT/dl-84/U6 $(\mathrm{GGAT})_{10}$.

The sequences of all mutant U6 promoter templates were verified by dideoxy sequencing. Purified plasmids were prepared by alkaline lysis of bacterial cells, $\mathrm{CsCl}$ gradient centrifugation, and chromatography on Bio-Gel A5m resin (Bio-Rad). DNA concentrations were determined spectrophotometrically by using $A_{260}$ and verified by visual examination of ethidium bromide-stained agarose gels.

Preparation of extracts for gel mobility shift analysis and in vitro transcription. The culturing of human 293 spinner cells and preparation of S100 extracts have been described previously (21). For some experiments, S100 extracts were prepared from HeLa spinner cells by identical methods. As an initial step to fractionate transcription factors and in order to deplete the extract of endogenous Oct- 1 protein, the S100 sample, dialyzed against buffer $\mathrm{D}(0.1 \mathrm{M} \mathrm{KCl}, 20 \mathrm{mM} N-2$ - hydroxyethylpiperazine- $N$ '-2-ethanesulfonic acid [HEPES; $\mathrm{pH}$ 7.9], 20\% glycerol, $0.2 \mathrm{mM}$ EDTA, $0.5 \mathrm{mM}$ dithiothreitol), was chromatographed over phosphocellulose (P11; Whatman) at a concentration of $10 \mathrm{mg}$ of protein per $\mathrm{ml}$ of resin equilibrated in buffer $D$. For in vitro transcription experiments, proteins were eluted with a single step of $0.6 \mathrm{M}$ KCl-20 mM HEPES (pH 7.9)-20\% glycerol-0.2 mM EDTA$0.5 \mathrm{mM}$ dithiothreitol and dialyzed against buffer D (P0.1-0.6 extract). The flowthrough $(0.1 \mathrm{M} \mathrm{KCl})$ included Oct- 1 and NONOCT factors, as determined by gel mobility shift analysis. Further fractionation of the NONOCT factor was achieved by chromatography of the phosphocellulose flowthrough sample over DEAE-cellulose (DE52; Whatman) at a concentration of $12 \mathrm{mg}$ of protein per $\mathrm{ml}$ of resin equilibrated in buffer $\mathrm{D}$. The protein fraction containing NONOCT factor was eluted from DE52 with buffer D containing $0.2 \mathrm{M} \mathrm{KCl}$ and dialyzed against buffer D (DE0.2 extract). Extracts were kept frozen at $-80^{\circ} \mathrm{C}$ until use. Protein concentration was determined by the Bradford method (5).

Purification of rOct-1 protein. Escherichia coli BL21(DE3) pLysS cells containing the human oct -1 cDNA (42) ligated into a pET vector (41) were supplied by the $D$. O. Peterson laboratory (Texas A\&M University). rOct-1 expressed from these cells contains 35 extra amino acids fused to the amino terminus that are encoded by a combination of the pET vector, the pBS M13+ vector (Stratagene) carrying the oct-1 insert, and the untranslated leader sequence of the oct-1 insert. Expression of rOct-1 was induced by the addition of isopropyl- $\beta$-D-thiogalactopyranoside to a concentration of 1 $\mathrm{mM}$ and incubation at $37^{\circ} \mathrm{C}$ for $3 \mathrm{~h}$. After centrifugation, the cell pellet was resuspended in 3 volumes of $50 \mathrm{mM}$ Tris $(\mathrm{pH}$ 7.9)-1 mM EDTA-12.5 mM $\mathrm{MgCl}_{2}-20 \%$ glycerol-0.5 M KCl-1 mM dithiothreitol. The cell suspension was treated sequentially with phenylmethylsulfonyl fluoride (final concentration of $0.5 \mathrm{mM}$ ) and lysozyme (final concentration of $0.5 \mathrm{mg} / \mathrm{ml}$ ), sodium deoxycholate (final concentration of $0.05 \%$ ), and DNase I (final concentration of $4.2 \mu \mathrm{g} / \mathrm{ml}$ ). After centrifugation $\left(12,000 \times \mathrm{g}, 15 \mathrm{~min}, 4^{\circ} \mathrm{C}\right)$, the pellet was washed with $1 \mathrm{M}$ urea-0.1 M Tris ( $\mathrm{pH} 8.5$ ), centrifuged again, and suspended in $4 \mathrm{ml}$ of $5 \mathrm{M}$ guanidine hydrochloride-50 mM Tris ( $\mathrm{pH}$ 7.9)-1 mM EDTA-12.5 mM $\mathrm{MgCl}_{2}-1$ $\mathrm{mM}$ dithiothreitol. Solubilized protein was diluted twice in a sequential manner with an equal volume of the buffer described above lacking guanidine hydrochloride (concentration reduced to $1.25 \mathrm{M}$ ) at room temperature and dialyzed overnight versus TM0.1 buffer (50 mM Tris [pH 7.9], $1 \mathrm{mM}$ EDTA, $12.5 \mathrm{mM} \mathrm{MgCl} 2,20 \%$ glycerol, $0.1 \mathrm{M} \mathrm{KCl}, 1 \mathrm{mM}$ dithiothreitol) at $4^{\circ} \mathrm{C}$. Oct- 1 protein was purified by chromatography over Sephacryl S200-HR equilibrated in TM0.1 buffer and OCTCON oligonucleotide DNA affinity chromatography. The affinity column matrix was prepared by using a 17-mer containing the U6 gene consensus octamer sequence (OCTCON; sequence given below) annealed and ligated as described previously (16) and affixed to Affi-Prep 10 activated resin (Bio-Rad) as described previously (49). Sephacryl fractions containing rOct-1 were pooled, adjusted to a final concentration of $9 \mu \mathrm{g}$ of poly $(\mathrm{dl}-\mathrm{dC})$ per $\mathrm{ml}$, and incubated with $1 \mathrm{ml}$ of packed OCTCON oligonucleotide resin for $1 \mathrm{~h}$ at $4^{\circ} \mathrm{C}$. The mixture was poured into a small column, washed with $20 \mathrm{mM}$ HEPES (pH 8.0)-20\% glycerol- $0.1 \%$ Nonidet $\mathrm{P}-40-1 \mathrm{mM}$ dithiothreitol- $0.1 \mathrm{M} \mathrm{KCl}$, and step eluted with the same buffer containing $0.2 \mathrm{M} \mathrm{KCl}, 0.4 \mathrm{M}$ $\mathrm{KCl}$, and $0.7 \mathrm{M} \mathrm{KCl}$. Oct-1 protein was eluted after addition of buffer containing $0.7 \mathrm{M} \mathrm{KCl}$ and dialyzed versus buffer $\mathrm{D}$ 
at $4^{\circ} \mathrm{C}$. The protein was stored at $-80^{\circ} \mathrm{C}$ in single-use aliquots.

DNase I footprinting. A singly end-labeled DNA fragment containing the U6 gene distal region was prepared via polymerase chain reaction amplification by standard procedures. The sequences of two oligonucleotides used for amplification (numbers denote the position in the human U6 gene $5^{\prime}$ flanking region) were -307 GCAAAACGCACC ACGTGACG -288 and -130 TTCTACGTCACGTATTTT GT -149 .

One oligonucleotide $(17 \mathrm{pmol})$ was labeled on the $5^{\prime}$ end with $\left[\gamma_{-}{ }^{32} \mathrm{P}\right] \mathrm{ATP}$ and T4 polynucleotide kinase and mixed with the other oligonucleotide (17 pmol) and a linearized U6 plasmid template (10 ng). Radiolabeled DNA fragments were purified by electrophoresis on polyacrylamide or agarose gels. For the footprinting reaction, approximately $3 \mathrm{fmol}$ $(20,000 \mathrm{dpm})$ of radiolabeled DNA fragment was mixed with various concentrations of rOct-1 in a total volume of $25 \mu \mathrm{l}$ containing $40 \mu \mathrm{g}$ of poly(dl-dC) per $\mathrm{ml}, 5 \mathrm{mM} \mathrm{MgCl}, 16 \mathrm{mM}$ HEPES (pH 7.9), $0.16 \mathrm{mM}$ EDTA, $16 \%$ glycerol, $80 \mathrm{mM}$ $\mathrm{KCl}$, and $0.8 \mathrm{mM}$ dithiothreitol for $30 \mathrm{~min}$ at $30^{\circ} \mathrm{C}$. Then $\mathrm{CaCl}_{2}$ was added to a final concentration of $0.1 \mathrm{mM}$, after which $1 \mu$ l of DNase I $(5 \mu \mathrm{g} / \mathrm{ml})$ was added immediately. After incubation for $2 \mathrm{~min}$ at room temperature, the enzyme digestion was stopped by the addition of EDTA to $9 \mathrm{mM}$, protein was removed by phenol- $\mathrm{CHCl}_{3}$ extraction, and DNA fragments were separated on $8 \%$ polyacrylamide-8.3 M urea gels and then subjected to autoradiography with an intensifying screen. Radiolabeled probes that had been cleaved partially by using the Maxam and Gilbert G-specific reaction (37) were used as electrophoresis markers.

Gel mobility shift assay. Radiolabeled DNA fragments were prepared by polymerase chain reaction using the primers listed above. To isolate a probe excluding the OCT2 and OCT3 sites, linearized OCT2MUT plasmid DNA was used as the template, and the amplified DNA fragment was restricted with KpnI enzyme to truncate within the disrupted OCT2 site prior to purification of the NONOCT/OCTCON fragment by gel electrophoresis. Approximately $3 \mathrm{fmol}$ of radiolabeled fragment was added to a binding mixture that contained $80 \mu \mathrm{g}$ of poly $(\mathrm{dl}-\mathrm{dC})$ per $\mathrm{ml}, 2 \mathrm{mM} \mathrm{MgCl}_{2}, 8 \mathrm{mM}$ HEPES (pH 7.9), 8\% glycerol, $0.08 \mathrm{mM}$ EDTA, $40 \mathrm{mM} \mathrm{KCl}$, and $0.4 \mathrm{mM}$ dithiothreitol. Proteins supplied by an S100 extract (approximately $2.5 \mu \mathrm{g}$ ) or the DE0.2 extract (approximately $6.5 \mu \mathrm{g}$ ) were added last, and the mixture was incubated for $30 \mathrm{~min}$ at $30^{\circ} \mathrm{C}$. Samples were electrophoresed on $4 \%$ polyacrylamide gels containing $6.7 \mathrm{mM}$ Tris-3.3 mM sodium acetate-1 mM EDTA ( $\mathrm{pH} 7.5)$ at room temperature. Gels were dried and autoradiographed with an intensifying screen. Double-stranded oligonucleotide competitors were annealed as described previously (16), diluted, and added to the binding reaction mixture prior to the protein as specified in the figures. The sequence of the top strand of each competitor DNA fragment (each end has a GATC $5^{\prime}$ overhang denoted by lowercase letters) was as follows: OCT CON, gatcCATATTTGCATAT; OCT2, gatcATAATTAGA ATTA; OCT3, gatcTTAATTTGACTGT; NONOCT, gatcC TATTTCCCATGATTC; NONOCTMUT, gatcCTtcTagag ATGATTC; and N.S. (nonspecific competitor), gatcCAGTC TGATCAGACTG.

Transfection experiments. Subconfluent monolayer 293 cells in 100-mm dishes were cotransfected with $10 \mu \mathrm{g}$ of a U6 maxigene plasmid DNA and $5 \mu \mathrm{g}$ of plasmid DNA containing the chicken $\beta$-tubulin gene (a control for transfection efficiency and RNA recovery), using the calcium phosphatemediated method described previously $(13,22)$. Total RNA

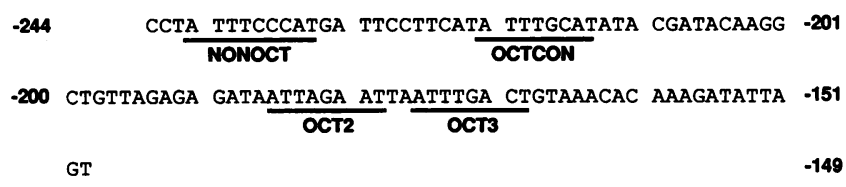

FIG. 1. Sequence of a human U6 snRNA gene distal region. The sequence of the top strand of a HaeIII-RsaI restriction fragment located from positions -244 to -149 upstream of the U6 transcription start site is shown. Various octamer-related sequences are underlined and described in the text.

was isolated 45 to $52 \mathrm{~h}$ posttransfection. Specific transcripts were detected by primer extension and polyacrylamide gel electrophoresis and quantitated by direct counting of the dried gel with a Betascope 603 blot analyzer (Betagen) as described previously (13).

In vitro transcription. $C$-free transcription reactions were carried out by mixing $200 \mathrm{ng}$ of plasmid DNA containing the $\mathrm{U} 6 /(\mathrm{GGAT})_{10}$ gene and $300 \mathrm{ng}$ of pGEM1 plasmid DNA with $10 \mu \mathrm{l}$ of phosphocellulose-fractionated S100 extract (P0.10.6 , a single-step elution from 0.1 to $0.6 \mathrm{M} \mathrm{KCl}$ as described earlier; approximately $12 \mu \mathrm{g}$ of protein), using conditions otherwise described previously (21). rOct-1 protein was diluted with buffer $D$ and added to some reactions just prior to the P0.1-0.6 extract. A constant amount of a radiolabeled, synthetic transcript prepared as described previously (21) was added to the stop mixture to control for variable recovery of RNA. Nucleic acids were separated on $10 \%$ polyacrylamide- $8.3 \mathrm{M}$ urea minigels $(6 \mathrm{~cm}$ in length) run in Tris-borate-EDTA buffer and visualized by autoradiography.

\section{RESULTS}

Multiple sites in a human U6 gene distal region are bound by Oct-1 protein in vitro. In previous work, the most significant stimulation of human U6 maxigene expression in transfected human cells was shown to originate from a restriction fragment spanning positions -244 to -149 of the 5 ' flanking region (22). [Since that publication, we have discovered that an extra T was mistakenly included in an oligo(dT) run of the sequence at -100 ; hence, the positions upstream are renumbered here.] The sequence of this fragment of DNA is shown in Fig. 1. In addition to the octamer motif (OCTCON), which had been noticed previously because of a perfect match to the consensus sequence, closer inspection revealed several other octamer-related sequences. Two of these sequences have a 6-of-8-bp match to the consensus and are referred to as OCT2 and OCT3. A third motif is upstream of the OCTCON element, and has a 7-of-8-bp match within a span of $9 \mathrm{bp}$. We have named this motif NONOCT.

To investigate the potential of the ubiquitous octamerbinding protein, Oct-1, to bind to these sites, we purified rOct-1 from $E$. coli and carried out a DNase I footprinting assay. As expected, the OCTCON motif was protected on both strands from DNase cleavage by the addition of rOct-1 (Fig. 2A). The protected region spanned approximately 20 bp. In addition, another area of protection was apparent that covered the OCT2/OCT 3 region. Interestingly, the 3 ' portion of OCT3 was not completely protected from DNase I cleavage. Indeed, the most complete bottom-strand footprint was centered over the OCT 2 motif, with only partial protection extending in the direction of OCT3 (Fig. 2A). Because the DNase I cleavage sites were relatively weak on naked DNA in the OCT2/OCT3 region, we could not determine the 


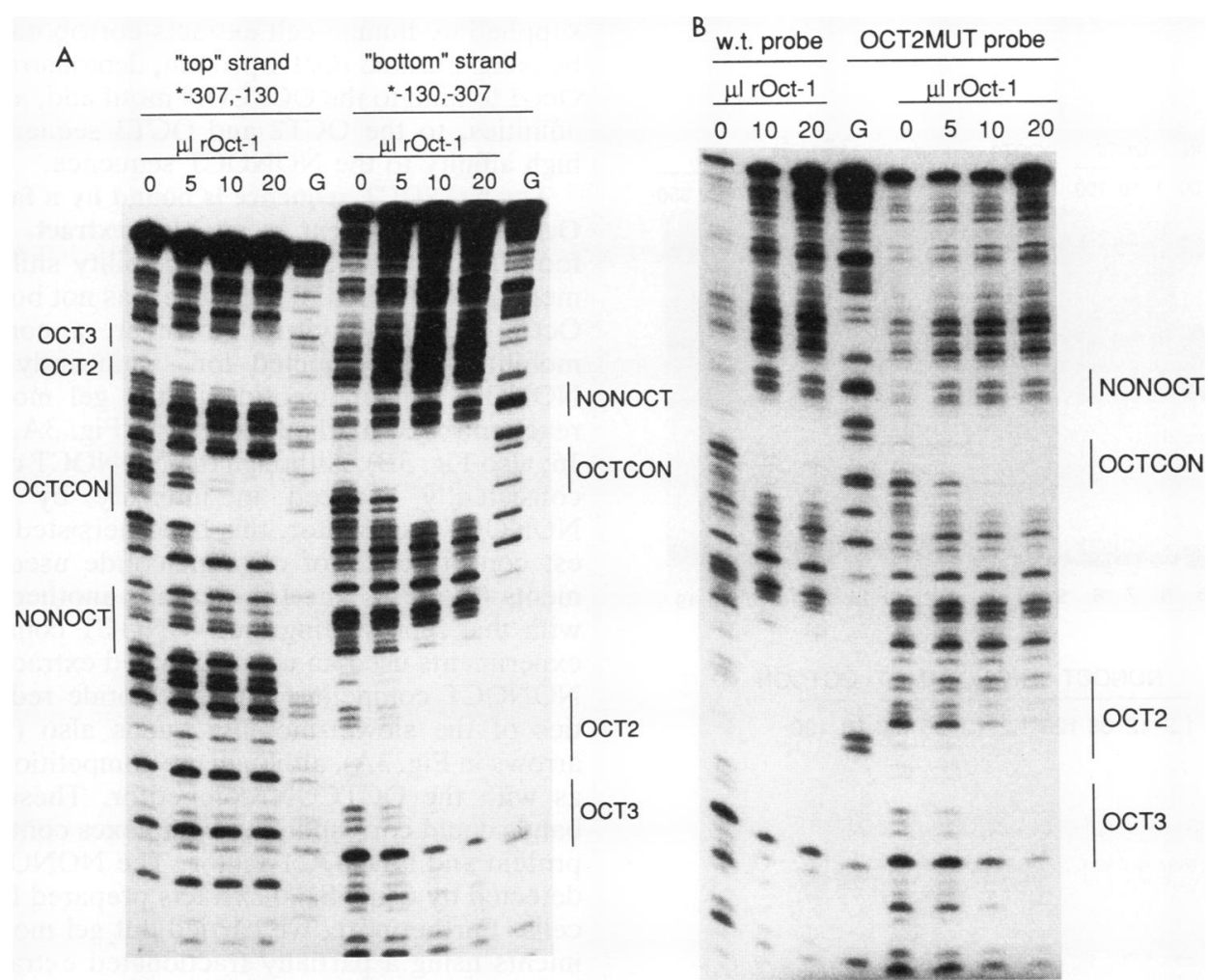

FIG. 2. DNase I protection analysis using recombinant Oct-1 protein with human U6 snRNA gene distal region probes. (A) Wild-type U6 gene DNA probes were prepared by the polymerase chain reaction, using end-labeled oligonucleotides corresponding to either strand. For each reaction, approximately $3 \mathrm{fmol}$ of DNA was incubated with the designated amount of rOct-1 protein, treated with DNase I, and electrophoresed on an $8 \%$ polyacrylamide sequencing gel. The concentration of the rOct-1 sample used for this experiment was approximately $0.15 \mathrm{mg} / \mathrm{ml}$. Top-strand samples were loaded early and thus electrophoresed farther into the gel. In the lanes marked G, samples of each probe were treated according to the Maxam and Gilbert G-specific reaction procedure to use as gel markers. (B) Bottom-strand probes for the wild-type (w.t.) U6 promoter and a template with a set of clustered point mutations in the OCT2 sequence (OCT2MUT) were end labeled at position -130 , using U6-specific primers in a polymerase chain reaction. Approximately 3 fmol of DNA was incubated with the indicated amount of rOct-1, treated with DNase I, and electrophoresed as described for panel A and in Materials and Methods.

precise extent of protection. When a set of clustered point mutations was introduced into the OCT2 sequence (OCT2MUT; sequence shown in Materials and Methods), we found a smaller region of protection by rOct-1, including only the $3^{\prime}$ half of the OCT2/OCT3 region (Fig. 2B). Thus, multiple Oct- 1 binding sites exist in the OCT2/OCT3 region, although the protected nucleotides appear to be displaced slightly with respect to the sequences corresponding to the octamer-like motifs. In contrast to the OCTCON, OCT2, and OCT3 sites, we could detect no protection over the NONOCT motif on either strand of DNA by this assay. We conclude that rOct-1 binds to the OCTCON motif and within the OCT2/OCT3 sequence but not to the NONOCT motif.

To determine whether octamer-binding protein present in a human cell extract could bind to these octamer-related sequences present in the U6 gene distal region, we used a gel mobility shift assay. A distal region DNA probe including wild-type sequence from -307 to -186 , and thus containing the NONOCT and OCTCON sites but lacking the OCT 2 and OCT3 sequences, was incubated with an $\mathrm{S} 100$ extract from human 293 cells. Several protein-DNA complexes were resolved on a polyacrylamide gel (Fig. 3A, lane 2). The major band (marked Oct) and at least two bands of slower mobility (marked with arrows) were competed for efficiently when a double-stranded oligonucleotide containing the consensus octamer motif was included in the binding reaction
(Fig. 3A, lanes 2 through 5). Therefore, it is likely that the major band corresponds to a complex of the relatively abundant Oct-1 protein with the U6 distal region probe. Indeed, a complex of nearly the same mobility was detected when rOct-1 protein was incubated with the probe and electrophoresed (results not shown). When double-stranded OCT 2 or OCT3 competitor oligonucleotide was added, competition of the octamer-related complexes was observed, although it was noticeably less efficient compared with the OCTCON competitor (Fig. 3A, lanes 6 through 11). The OCT3 oligonucleotide was a somewhat better competitor than the OCT2 oligonucleotide. In agreement with the inability of rOct-1 to bind specifically to the NONOCT sequence in a footprinting assay, excess NONOCT oligonucleotide did not compete efficiently for the formation of the major octamer-binding protein-DNA complex (Fig. 3A, lanes 13 through 16). Significantly, a large molar excess of a nonspecific competitor oligonucleotide did not reduce the intensity of any bands, except at the highest concentration used, where the intensities of all bands were reduced slightly (Fig. $3 \mathrm{~A}$, lanes 17 through 19). At very high concentrations of NONOCT oligonucleotide, we did notice some reduction of the octamer protein-DNA complex in most experiments (Fig. 3A, lane 16; Fig. 3B, lane 6). It is possible that Oct-1 has a greater affinity for the NONOCT sequence than for random DNA. Thus, results obtained by using proteins 

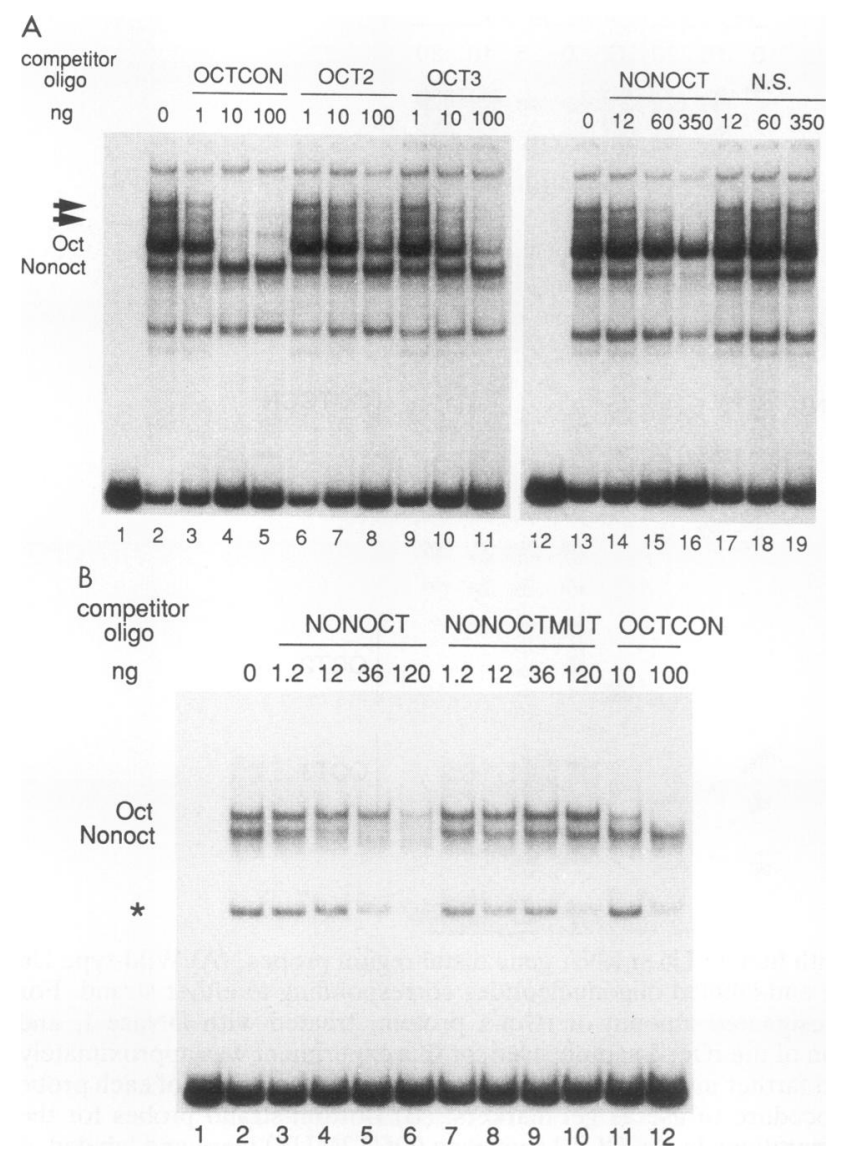

FIG. 3. Gel mobility shift analysis of protein-DNA complexes assembled on the U6 gene distal region. (A) A DNA probe containing wild-type NONOCT and OCTCON sequences was prepared by using an end-labeled U6-specific top-strand oligonucleotide and an unlabeled bottom-strand oligonucleotide by polymerase chain reaction amplification using an OCT2MUT template. The probe was truncated by KpnI restriction within the mutated OCT2 sequence and purified by gel electrophoresis. Approximately $3 \mathrm{fmol}$ of DNA was incubated with approximately $2.5 \mu \mathrm{g}$ of S100 extract protein from 293 cells and loaded directly on a $4 \%$ polyacrylamide gel in Tris-acetate-EDTA buffer. Double-stranded oligonucleotide competitor DNAs were added prior to addition of the extract as indicated above the lanes. One, 10, and $100 \mathrm{ng}$ of OCTCON, OCT2, and OCT 3 oligonucleotides correspond to approximately 30-, 300-, and 3,000-fold molar excesses compared to the radiolabeled probe. Twelve, 60, and $350 \mathrm{ng}$ of NONOCT and N.S. (nonspecific) oligonucleotide correspond to approximately $300-, 1,500$-, and 9,000-fold molar ratios. (B) Another gel mobility shift assay using a partially fractionated HeLa cell extract enriched in NONOCT factor. Approximately $3 \mathrm{fmol}$ of radiolabeled probe DNA containing the OCTCON and NONOCT sites was mixed with the amount of unlabeled competitor oligonucleotide noted above each lane and incubated with approximately $6.5 \mu \mathrm{g}$ of DE0.2 extract prepared as described in Materials and Methods. Samples were electrophoresed as described for panel $A$ and in Materials and Methods. NONOCT, NONOCTMUT, and OCTCON competitor DNAs were added prior to the addition of protein at molar ratios of 30- to 3,000-fold excess compared with the radiolabeled probe. The band marked with the asterisk represents an unidentified complex that is competed for efficiently by all three oligonucleotides at the highest amounts tested. supplied by human cell extracts corroborate those obtained by using purified rOct- 1 protein, demonstrating the ability of Oct- 1 to bind to the OCTCON motif and, at somewhat lower affinities, to the OCT2 and OCT3 sequences but not with high affinity to the NONOCT sequence.

The NONOCT sequence is bound by a factor distinct from Oct-1 that is present in an $\mathbf{S 1 0 0}$ extract. As shown in the footprinting (Fig. 2) and gel mobility shift (Fig. 3) experiments, the NONOCT sequence was not bound efficiently by Oct-1 protein in vitro. However, a complex of greater mobility was competed for significantly by inclusion of NONOCT oligonucleotide in the gel mobility shift assay reaction (band marked Nonoct in Fig. 3A, lanes 13 through 16; also Fig. 3B). Although the NONOCT complex band was consistently reduced in intensity by addition of the NONOCT competitor, this band persisted even at the highest concentration of oligonucleotide used in most experiments (Fig. 3A, lane 16). Perhaps another band comigrated with that representing the NONOCT complex, since these experiments used an unfractionated extract. Addition of the NONOCT competitor oligonucleotide reduced the intensities of the slower-mobility bands also (marked with the arrows in Fig. 3A), although the competition was not as great as with the OCTCON competitor. These slower-mobility bands could correspond to complexes containing both Oct-1 protein and NONOCT factor. The NONOCT complex was detected by using S100 extracts prepared from 293 or HeLa cells. Furthermore, we carried out gel mobility shift experiments using a partially fractionated extract preparation in which the NONOCT factor was enriched relative to Oct-1 by chromatography of the S100 extract over phosphocellulose and DEAE-cellulose columns. Using this extract, Oct and NONOCT complexes of approximately equal intensities are detected after gel electrophoresis (Fig. 3B, lane 2). The NONOCT complex is competed for by addition of the NONOCT oligonucleotide, whereas its presence is unchanged by the addition of an oligonucleotide with clustered mutations in this element (NONOCTMUT) or by the OCT CON oligonucleotide (Fig. 3B; compare lanes 3 through 5 with lanes 7 through 10 and lanes 11 and 12). As noted above, at very high concentrations of NONOCT oligonucleotide, the Oct-1 complex is reduced (Fig. 3B, lane 6). Furthermore, an unidentified complex marked with the asterisk is competed for by all three oligonucleotide competitors in this experiment and is therefore unlikely to represent formation of a sequence-specific protein-DNA complex (Fig. $3 \mathrm{~B}$, lanes 6,10 , and 12 ). We conclude that a factor present in these extracts, which is distinct from Oct-1, recognizes a sequence in the NONOCT oligonucleotide.

The OCTCON and NONOCT sequences are functional elements in the human $U 6$ distal region. To determine whether the consensus and nonconsensus octamer sequences are transcriptionally active in the context of the human U6 gene distal region, we constructed a set of plasmid DNA templates in which these sites were disrupted by clustered point mutations within specific elements or in various combinations of mutated elements in a U6 maxigene (Fig. 4A). Since the precise extents of the factor binding sites within the OCT2/OCT3 and NONOCT regions were not determined, each mutant template contained six nucleotide changes relative to the normal sequence of each element. Indeed, the DNase I footprints (Fig. 2) indicated a slight incongruence of the protected nucleotides relative to the OCT2 and OCT3 sequences. Each plasmid DNA was introduced into human 293 cells by using a calcium phosphatemediated transient expression transfection assay. Total 

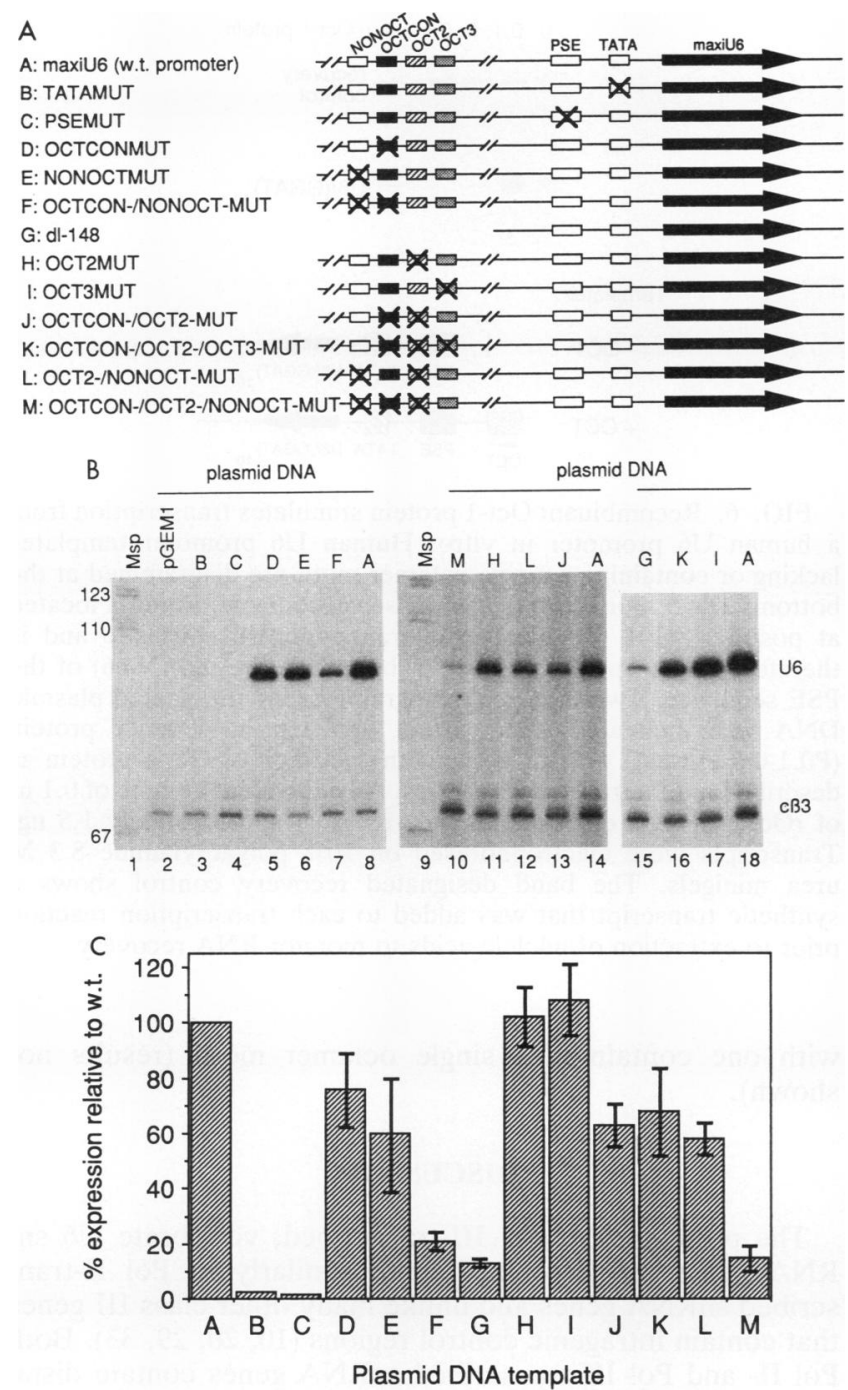

FIG. 4. Transient expression of U6 maxigene templates containing disrupted distal region elements after transfection of human 293 cells. (A) Diagram of U6 maxigene plasmid DNA templates used for the transfection experiments. A single letter from $A$ to $M$ is used to denote each template in panels B and C. w.t., wild type. (B) Typical results of U6 maxigene expression in transfected cells as detected by primer extension analysis and electrophoresis on $10 \%$ polyacrylamide sequencing gels. Each panel shows the results from different experiments. U6 represents the primer extension product from U6 maxigene RNA, and $c \beta 3$ represents a set of bands corresponding to transcripts initiated from a chicken $\beta$-tubulin gene contained in a cotransfected plasmid used as a control to monitor variable efficiency of transfection and RNA recovery. The lanes marked Msp are radiolabeled DNA fragments from MspI digestion of pGEM1 plasmid DNA used as gel electrophoresis markers. Sizes are indicated in nucleotides. (C) Quantitation of transfection experiments. The results originating from at least three separate dishes of transfected cells were included in the analysis except for the TATAMUT (B) and PSEMUT (C) templates. Primer extension products that had been electrophoresed on polyacrylamide gels were quantitated with a Betagen Betascope 603 blot analyzer. After background subtraction, the level of each U6 band was normalized according to the cB3 band intensity in that lane and compared with that from a wild-type (w.t.) U6 maxigene template included in each experiment. The height of each column represents the average value, and the error bar shows 1 standard deviation from the mean.
RNA was isolated approximately 2 days after addition of the DNA, and U6 maxigene-specific transcripts were detected by primer extension and polyacrylamide gel electrophoresis. A plasmid that carried a chicken $\beta$-tubulin gene was included in each sample as a control for transfection efficiency and RNA recovery. Typical primer extension assay results from various experiments are shown in Fig. 4B. Each distal region mutant template was tested in at least three independent transfections, and the transcriptional signal relative to that of the wild-type promoter was quantitated (Fig. 4C). In these experiments, the presence of the distal region stimulated U6 gene transcription approximately 10-fold (compare template G [dl-148] with template A [wild type]). In comparison, disruption of a proximal sequence element, the TATA element (template B) or the PSE (template C), resulted in a more severe drop in transcriptional efficiency (approximately 50-fold). Disruption of the consensus octamer motif resulted in only a small reduction of U6 gene expression (template D [OCTCONMUT]). When the OCT2 and OCT3 nonconsensus octamer motifs were mutated individually, expression remained at wild-type levels (templates $\mathbf{H}$ [OCT2MUT] and I [OCT3MUT]). Even when all three sites that bound Oct-1 protein in vitro were disrupted in the same plasmid (template $\mathbf{K}$ ), the transcriptional signal was not significantly lower than when the consensus octamer motif alone was mutated. Hence, the presence of the OCT2 and OCT3 sites was insignificant in the transient expression assay.

Disruption of the NONOCT sequence alone resulted in a moderate reduction of U6 maxigene expression (Fig. 4, template E [NONOCTMUT]). The decrease in transcription was consistently greater for a template with a mutated NONOCT sequence (template E) than for one with a disrupted OCTCON motif (template D), although this difference was very minor. Significantly, when the NONOCT and OC TCON sequences were disrupted in the same template, distal region function was eliminated almost completely (template $F$ [OCTCON-/NONOCT-MUT]). Mutations within NONOCT and OCT2 in the same plasmid (template L) resulted in only a moderate reduction reminiscent of the NONOCTMUT signal (template E), demonstrating again the insignificant role of the OCT2 sequence in vivo. These transfection results indicate functional roles for both the NONOCT and OCTCON sequences.

The presence of a NONOCT sequence is not required for maximal transcriptional activation by the distal region. Introduction of clustered point mutations within the NONOCT sequence reduced U6 maxigene expression to approximately $60 \%$ of the wild-type level (Fig. 4, template E; Fig. 5, lane 4), but this level of transcription was significantly above that from a promoter lacking the distal region entirely. Therefore, the NONOCT sequence is not absolutely required for some degree of transcriptional activation. To determine whether the maximal level of expression could be obtained without the NONOCT site, we converted it to a consensus octamer sequence by site-directed mutagenesis (CONVROCT template; sequence shown in Materials and Methods). Expression from the CONVROCT template was at wild-type levels in transfected 293 cells (Fig. 5; compare lanes 5 and 6). The average result from three separate experiments was $110 \%$ ( \pm 18 [1 standard deviation]) compared with that of a wildtype promoter. Thus, in the absence of the NONOCT sequence, two rather closely spaced consensus octamer motifs at the normal distal region location can act as a potent transcriptional activator.

rOct-1 protein can stimulate human U6 gene transcription 


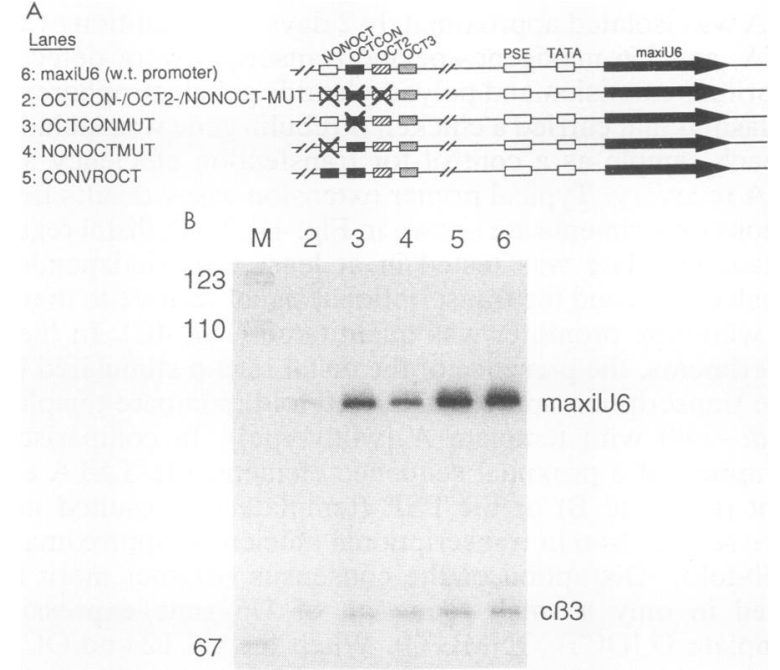

FIG. 5. Conversion of the NONOCT element to a consensus octamer motif results in wild-type U6 maxigene expression in transfected 293 cells. (A) Diagram of U6 maxigene templates used for the transfection experiment shown in panel B. w.t., wild type. (B) U6 maxigene expression from mutant or wild-type templates shown in panel A. Following transfection of 293 cells, total RNA was isolated from each dish, and specific transcripts were detected by primer extension analysis. maxiU6 denotes the U6 maxigene transcript primer extension product, and $c \beta 3$ denotes primer extension products from the cotransfected chicken $\beta$-tubulin gene plasmid added to each dish as a control for transfection efficiency and RNA recovery. The lane marked $M$ contains radiolabeled DNA markers from an MspI restriction digest of pGEM1 plasmid DNA. Sizes are indicated in nucleotides.

in vitro. Purified Oct-1 and Oct- 2 transcription factors have been shown to stimulate transcription by RNA Pol III from a human 7 SK gene promoter $(30,31)$. Previously, in vitro transcription from U6 genes in crude S100 extracts has demonstrated only a minor role, if any, of distal sequences $(12,21,22,47)$. However, a recent report demonstrated that the close positioning of an octamer binding site and the 7SK gene PSE potentiated the binding of PSE transcription factor and resulted in significant activation by Oct-1 protein (31). Therefore, we constructed a human U6 plasmid containing a C-free cassette (21) with a single OCTCON sequence at a location adjacent to the proximal promoter (Fig. 6, +OCT template; the legend provides details regarding the spacing between the PSE and octamer sites). Transcription was carried out in a 293 cell S100 extract chromatographed on phosphocellulose to effect depletion of endogenous Oct-1 protein. Gel mobility shift analyses indicated that both Oct-1 and NONOCT factor did not bind to phosphocellulose under the conditions used $(0.1 \mathrm{M} \mathrm{KCl})$, so the transcription extract was depleted in NONOCT factor also (results not shown). Addition of approximately $1.5 \mathrm{ng}$ of rOct-1 to the depleted extract with the +OCT template stimulated transcription five- to sixfold (Fig. 6). In other experiments, we found that the level of transcriptional stimulation varied from approximately 3 - to 10 -fold when a comparable mass of rOct-1 was included in the reactions. Addition of the same amount of rOct-1 to a reaction with a U6 promoter lacking an octamer motif resulted in no transcriptional stimulation (Fig. 6, -OCT template). The relative ability to activate transcription was unchanged when a U6 proximal promoter template containing two copies of the octamer motif was compared

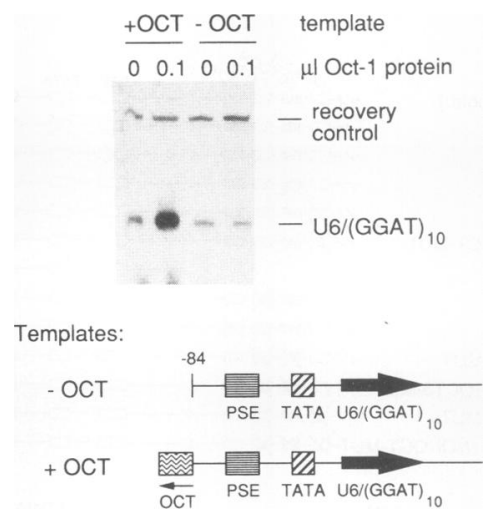

FIG. 6. Recombinant Oct-1 protein stimulates transcription from a human U6 promoter in vitro. Human U6 promoter templates lacking or containing a single octamer motif are diagrammed at the bottom. The $5^{\prime}$ boundary of the consensus octamer motif is located at position -101 relative to the transcriptional start site and is therefore $35 \mathrm{bp}$ upstream of the $5^{\prime}$ boundary (position -66 ) of the PSE sequence. Two hundred nanograms of the designated plasmid DNA was incubated with extract depleted in octamer protein (P0.1-0.6 extract) with or without the addition of Oct-1 protein as described in Materials and Methods. An equivalent volume of $0.1 \mu \mathrm{l}$ of rOct-1 protein corresponds to a mass of approximately $1.5 \mathrm{ng}$. Transcripts were electrophoresed on $10 \%$ polyacrylamide-8.3 M urea minigels. The band designated recovery control shows a synthetic transcript that was added to each transcription reaction prior to extraction of nucleic acids to monitor RNA recovery.

with one containing a single octamer motif (results not shown).

\section{DISCUSSION}

The promoters of Pol III-transcribed, vertebrate U6 snRNA genes are organized quite similarly to Pol II-transcribed snRNA genes and unlike many other class III genes that contain intragenic control regions $(10,20,29,33)$. Both Pol II- and Pol III-transcribed snRNA genes contain distal sequence elements greater than $200 \mathrm{bp}$ upstream from the transcriptional start site. We have identified two elements in the distal region of a human U6 snRNA gene that are necessary for full transcriptional activation in transfected 293 cells. One element is the previously characterized octamer motif that is bound by Oct-1 protein in vitro $(4,6)$. The other element is not recognized by Oct-1 with high affinity, and we refer to it as NONOCT.

The presence of two functional elements in the human U6 distal region, one of which is an octamer motif, is a characteristic shared by snRNA genes that are transcribed by RNA Pol II. We have not delineated yet the precise boundaries of the second element. At least a portion of that site must be located within the set of $6 \mathrm{bp}$ that were mutated to give the NONOCTMUT template. The same set of mutations contained in the NONOCTMUT template also eliminated the ability to compete for binding of a NONOCT factor detected in cultured cell extracts. Repeated attempts to obtain a distinct footprint over the NONOCT region using crude extracts have been unsuccessful so far.

In addition to an octamer motif, several other transcriptional control elements have been identified as part of snRNA gene distal regions. The well-characterized Sp1 transcription factor binds to sites in the human and Xenopus $\mathrm{U} 2$ and the Xenopus U6 gene distal regions $(2,14,25,45)$. 
The NONOCT sequence does not conform to a consensus Spl binding site and therefore is not likely to be recognized by this transcription factor. However, an activator role for Sp1 in the human U6 gene should not be excluded, since a GC-rich sequence (CCGCGCGCCG) exists between positions -279 and -270 , and previous transfection experiments have demonstrated a moderate stimulatory effect of sequences upstream from position - 244 (22). The mouse U6 gene distal region contains potential $\mathrm{Sp} 1$ binding sites also (12). Another control element, the SPH motif, has been identified within the distal regions of several vertebrate snRNA genes $(7,35,36,50)$ and the Xenopus tRNA ${ }^{\text {(Ser)Sec }} 5^{\prime}$ flanking region (32). However, the extended NONOCT sequence has a very poor fit to these snRNA gene SPH motifs, and therefore we consider it unlikely that the same transcription factor interacts with these control elements. Other motifs found in Pol II- or Pol III-transcribed snRNA gene distal regions, such as a CACCC box (19), an AP-2 binding site (48), and a cAMP response element (48), appear to be distinct from any sequence in the vicinity of NONOCT. Although the NONOCT CCCAT sequence is reminiscent of the CCAAT core sequence found in vertebrate U3 gene enhancers $(1,38)$, the completely conserved U3-box hexanucleotide (CCAATC) is not a perfect match. Therefore, it is likely that the NONOCT element in the human U6 distal region is different from those identified previously. Furthermore, no similar sequence is located in close proximity to the octamer motifs within the mouse or Xenopus U6 distal regions either. Whether or not the NONOCT control element is conserved among human U6 genes is not known and awaits isolation of more human U6 true genes.

The NONOCT element does not appear to be required for transcriptional stimulation. U6 maxigene expression from the NONOCTMUT template is elevated over that from a distalless promoter in transfection experiments. Furthermore, the substitution of the NONOCT sequence with a consensus octamer motif results in wild-type expression. These results with a human U6 gene distal region are similar to those found with a human 7SK gene in that the natural enhancer-like region is composed of an octamer motif (imperfect in the 7SK gene promoter) and at least one other element that is not bound by Oct-1. In the 7SK distal region, this second element contains a CACCC sequence (19). However, with both genes, full stimulatory activity can be attained with the inclusion of one or two copies of a consensus octamer motif in the absence of the CACCC element or the NONOCT element (references 18 and 31 and this study).

Clearly, our experiments and previous results for the human 7SK gene promoter demonstrate that purified Oct-1 can stimulate Pol III transcriptional levels from these promoters (30, $31)$. Those investigators also found that Oct-1, Oct-2, or the conserved POU domain of these proteins could potentiate the binding of a transcription factor that recognized the snRNA gene PSE (31). Thus, it was hypothesized that activation of snRNA transcription by octamer-binding protein could be achieved by promoting the binding of PSE transcription factor. We are purifying the NONOCT factor to determine whether this protein can stimulate transcription in vitro in the absence of octamer-binding protein or whether it acts only in concert with Oct-1. We have not detected transcriptional stimulation from a U6 promoter template with a single ligated copy of the NONOCT sequence in a 293 cell S100 extract.

In addition to the consensus octamer motif, we detected at least two other sequences in the human U6 gene distal region that were bound specifically by Oct-1 in vitro. However disruption of either the OCT2 or the OCT3 site individually resulted in no effect on U6 maxigene expression in vivo. Furthermore, a plasmid template containing clustered mutations that destroy all of the sites recognized by Oct- 1 within the region examined here (OCTCON, OCT2, and OCT3 sites) was transcribed at a level not significantly below that of a plasmid with only a disrupted consensus octamer motif. Therefore, results from our transfection experiments discount any significant roles for the nonconsensus Oct-1 binding sites. It is quite possible that these results are explained by differential affinity of Oct-1 among these various binding sites. Indeed, the individual OCT2 or OCT3 sequences were poorer competitors in gel mobility shift experiments than was the consensus octamer sequence. However, this interpretation is not entirely consistent when the DNase footprinting experiments are considered. We detected little difference in the degree of protection over these various sites as the Oct-1 protein concentration was increased. Another possible explanation to account for the absence of any role of the OCT 2 and OCT 3 sites in vivo is that these sites are blocked by a specific chromatin configuration and therefore rendered inoperative. Perhaps a phased nucleosome covers the OCT2/OCT3 region while leaving the NONOCT and OCTCON sites available for binding by their requisite transcription factors. Further analysis of the structure of Pol III gene enhancer-like regions will yield important insight into the mechanisms employed to activate transcription from eukaryotic promoters.

\section{ACKNOWLEDGMENTS}

We thank the D. O. Peterson laboratory (Texas A\&M University) for supplying the cells containing human oct-1 cDNA (originally isolated by the W. Herr laboratory, Cold Spring Harbor Laboratory) inserted into a pET vector and for assisting with purification procedures. Paula Middleton constructed some of the recombinant plasmids used for the transfection experiments. Also we acknowledge the assistance of Ruth White for the maintenance of human 293 cell spinner cultures and the purification of several plasmid DNAs used in these experiments.

This work was supported by NSF grant DMB-8903970.

\section{REFERENCES}

1. Ach, R. A., and A. M. Weiner. 1991. Cooperation between CCAAT and octamer motifs in the distal sequence element of the rat U3 small nucleolar RNA promoter. Nucleic Acids Res. 19:4209-4218.

2. Ares, M., Jr., J.-S. Chung, L. Giglio, and A. M. Weiner. 1987. Distinct factors with Spl and NF-A specificities bind to adjacent functional elements of the human U2 snRNA gene enhancer. Genes Dev. 1:808-817.

3. Ares, M., Jr., M. Mangin, and A. M. Weiner. 1985. Orientationdependent transcriptional activator upstream of a human U2 snRNA Gene. Mol. Cell. Biol. 5:1560-1570.

4. Bark, C., P. Weller, J. Zabielski, L. Janson, and U. Pettersson. 1987. A distant enhancer element is required for polymerase III transcription of a U6 RNA gene. Nature (London) 328:356-359.

5. Bradford, M. M. 1976. A rapid and sensitive method for the quantitation of microgram quantities of protein utilizing the principle of protein-dye binding. Anal. Biochem. 72:248-254.

6. Carbon, P., S. Murgo, J.-P. Ebel, A. Krol, G. Tebb, and I. W. Mattaj. 1987. A common octamer motif binding protein is involved in the transcription of U6 snRNA by RNA polymerase III and U2 snRNA by RNA polymerase II. Cell 51:71-79.

7. Cheung, C. H., Q. N. Fan, and W. E. Stumph. 1993. Structural requirements for the functional activity of a U1 snRNA gene enhancer. Nucleic Acids Res. 21:281-287.

8. Ciliberto, G., F. Palla, G. Tebb, I. W. Mattaj, and L. Philipson. 1987. Properties of a U1 enhancer-like sequence. Nucleic Acids Res. 15:2403-2416.

9. Dahlberg, J. E., and E. Lund. 1988. The genes and transcription 
of the major small nuclear RNAs, p. 38-70. In M. L. Birnstiel (ed.), Structure and function of major and minor small nuclear ribonucleoprotein particles. Springer-Verlag, Berlin.

10. Dahlberg, J. E., and E. Lund. 1991. How does III $\times$ II make U6. Science 254:1462-1463.

11. Dahlberg, J. E., and E. T. Schenborn. 1988. The human U1 snRNA promoter and enhancer do not direct synthesis of messenger RNA. Nucleic Acids Res. 16:5827-5840.

12. Das, G., D. Henning, D. Wright, and R. Reddy. 1988. Upstream regulatory elements are necessary and sufficient for transcription of a U6 RNA gene by RNA polymerase III. EMBO J. 7:503-512.

13. Goomer, R. S., and G. R. Kunkel. 1992. The transcriptional start site for a human U6 small nuclear RNA gene is dictated by a compound promoter element consisting of the PSE and the TATA box. Nucleic Acids Res. 20:4903-4912.

14. Janson, L., C. Bark, and U. Pettersson. 1987. Identification of proteins interacting with the enhancer of human U2 small nuclear RNA genes. Nucleic Acids Res. 15:4997-5016.

15. Janson, L., and U. Pettersson. 1990. Cooperative interactions between transcription factors Sp1 and OTF-1. Proc. Natl. Acad. Sci. USA 87:4732-4736.

16. Kadonaga, J. T., and R. Tjian. 1986. Affinity purification of sequence-specific DNA binding proteins. Proc. Natl. Acad. Sci. USA 83:5889-5893.

17. Kazmaier, M., G. Tebb, and I. W. Mattaj. 1987. Functional characterization of $X$. laevis U5 snRNA genes. EMBO J. 6:3071-3078.

18. Kleinert, H., R. Assert, and B.-J. Benecke. 1991. A single base pair deletion from the inactive octamer-like motif of the 7S K distal sequence element brings full functionality in vivo. J. Biol. Chem. 266:23872-23877.

19. Kleinert, H., S. Bredow, and B.-J. Benecke. 1990. Expression of a human 7SK RNA gene in vivo requires a novel pol III upstream element. EMBO J. 9:711-718.

20. Kunkel, G. R. 1991. RNA polymerase III transcription of genes that lack internal control regions. Biochim. Biophys. Acta 1088:1-9.

21. Kunkel, G. R., and D. A. Danzeiser. 1992. Formation of a template committed complex on the promoter of a gene for the U6 small nuclear RNA from the human requires multiple sequence elements, including the distal region. J. Biol. Chem. 267:14250-14258.

22. Kunkel, G. R., and T. Pederson. 1988. Upstream elements required for efficient transcription of a human U6 RNA gene resemble those of $U 1$ and $U 2$ genes even though a different polymerase is used. Genes Dev. 2:196-204.

23. Kunkel, G. R., and T. Pederson. 1989. Transcription of a human U6 small nuclear RNA gene in vivo withstands deletion of intragenic sequences but not of an upstream TATATA box. Nucleic Acids Res. 17:7371-7379.

24. Kunkel, T. A. 1985. Rapid and efficient site-specific mutagenesis without phenotypic selection. Proc. Natl. Acad. Sci. USA 82:488-492.

25. Lescure, A., G. Tebb, I. W. Mattaj, A. Krol, and P. Carbon. 1992. A factor with Spl DNA-binding specificity stimulates Xenopus U6 snRNA in vivo transcription by RNA polymerase III. J. Mol. Biol. 228:387-394.

26. Lobo, S. M., J. Lister, M. L. Sullivan, and N. Hernandez. 1991. The cloned RNA polymerase II transcription factor IID selects RNA polymerase III to transcribe the human U6 gene in vitro. Genes Dev. 5:1477-1489.

27. Mattaj, I. W., S. Lienhard, J. Jiricny, and E. M. De Robertis. 1985. An enhancer-like sequence within the Xenopus U2 gene promoter facilitates the formation of stable transcription complexes. Nature (London) 316:163-167.

28. Murphy, S., C. Di Liegro, and M. Melli. 1987. The in vitro transcription of the 7SK RNA gene by RNA polymerase III is dependent only on the presence of an upstream promoter. Cell 51:81-87.

29. Murphy, S., B. Moorefield, and T. Pieler. 1989. Common mechanisms of promoter recognition by RNA polymerases II and III. Trends Genet. 5:122-126.
30. Murphy, S., A. Pierani, C. Scheidereit, M. Melli, and R. G. Roeder. 1989. Purified octamer binding transcription factors stimulate RNA polymerase III-mediated transcription of the 7SK RNA gene. Cell 59:1071-1080.

31. Murphy, S., J.-B. Yon, T. Gerster, and R. G. Roeder. 1992. Oct-1 and Oct-2 potentiate functional interactions of a transcription factor with the proximal sequence element of small nuclear RNA genes. Mol. Cell. Biol. 12:3247-3261.

32. Myslinski, E., A. Krol, and P. Carbon. 1992. Optimal tRNA(Ser) Sec gene activity requires an upstream SPH motif. Nucleic Acids Res. 20:203-209.

33. Palmer, J. M., and W. R. Folk. 1990. Unraveling the complexities of transcription by RNA polymerase III. Trends Biochem. Sci. 15:300-304.

34. Reddy, R. 1988. Transcription of a U6 small nuclear RNA gene in vitro. Transcription of a mouse U6 small nuclear RNA gene in vitro by RNA polymerase III is dependent on transcription factor(s) different from transcription factors IIIA, IIIB, and IIIC. J. Biol. Chem. 263:15980-15984.

35. Roebuck, K. A., D. P. Szeto, K. P. Green, Q. N. Fan, and W. E. Stumph. 1990. Octamer and SPH motifs in the U1 enhancer cooperate to activate U1 RNA gene expression. Mol. Cell. Biol. 10:341-352.

36. Roebuck, K. A., R. J. Walker, and W. E. Stumph. 1987. Multiple functional motifs in the chicken U1 RNA gene enhancer. Mol. Cell. Biol. 7:4185-4193.

37. Sambrook, J., E. F. Fritsch, and T. Maniatis. 1989. Molecular cloning: a laboratory manual, 2nd ed. Cold Spring Harbor Laboratory Press, Cold Spring Harbor, N.Y.

38. Savino, R., Y. Hitti, and S. A. Gerbi. 1992. Genes for Xenopus laevis U3 small nuclear RNA. Nucleic Acids Res. 20:5435-5442.

39. Simmen, K. A., J. Bernues, H. D. Parry, H. G. Stunnenberg, A. Berkenstam, B. Cavallini, J.-M. Egly, and I. W. Mattaj. 1991. TFIID is required for in vitro transcription of the human U6 gene by RNA polymerase III. EMBO J. 10:1853-1862.

40. Skuzeski, J. M., E. Lund, J. T. Murphy, T. H. Steinberg, R. R. Burgess, and J. E. Dahlberg. 1984. Synthesis of human U1 RNA. II. Identification of two regions of the promoter essential for transcription initiation at position +1 . J. Biol. Chem. 259: 8345-8352.

41. Studier, F. W., A. H. Rosenberg, J. J. Dunn, and J. W. Dubendorf. 1990. Use of T7 RNA polymerase to direct expression of cloned genes. Methods Enzymol. 185:60-89.

42. Sturm, R. A., G. Das, and W. Herr. 1988. The ubiquitous octamer-binding protein Oct-1 contains a POU domain with a homeo box subdomain. Genes Dev. 2:1582-1599.

43. Tanaka, M., U. Grossniklaus, W. Herr, and N. Hernandez. 1988. Activation of the U2 snRNA promoter by the octamer motif defines a new class of RNA polymerase II enhancer elements. Genes Dev. 2:1764-1778.

44. Tanaka, M., J.-S. Lai, and W. Herr. 1992. Promoter-selective activation domains in Oct-1 and Oct-2 direct differential activation of an snRNA and mRNA promoter. Cell 68:755-767.

45. Tebb, G., and I. W. Mattaj. 1989. The Xenopus laevis U2 gene distal sequence element (enhancer) is composed of four subdomains that can act independently and are partly functionally redundant. Mol. Cell. Biol. 9:1682-1690.

46. Waldschmidt, R., and K. H. Seifart. 1992. TFIIA is required for in vitro transcription of mammalian U6 genes by RNA polymerase III. J. Biol. Chem. 267:16359-16364.

47. Waldschmidt, R., I. Wanandi, and K. H. Seifart. 1991. Identification of transcription factors required for the expression of mammalian U6 genes in vitro. EMBO J. 10:2595-2603.

48. Weller, P., C. Bark, L. Janson, and U. Pettersson. 1988. Transcription analysis of a human U4C gene: involvement of transcription factors novel to snRNA gene expression. Genes Dev. 2:1389-1399.

49. Yoshinaga, S. K., N. D. L'Etoile, and A. J. Berk. 1989. Purification and characterization of transcription factor IIIC2. J. Biol. Chem. 264:10726-10731.

50. Zamrod, Z., and W. E. Stumph. 1990. U4B snRNA gene enhancer activity requires functional octamer and SPH motifs. Nucleic Acids Res. 18:7323-7330. 